\title{
Headspace passive dosing of volatile hydrophobic chemicals - Aquatic toxicity testing exactly at the saturation level
}

Trac, Lam Ngoc; Nørgaard Schmidt, Stine; Mayer, Philipp

Published in:

Chemosphere

Link to article, DOI:

10.1016/j.chemosphere.2018.07.150

Publication date:

2018

Document Version

Peer reviewed version

Link back to DTU Orbit

Citation (APA):

Trac, L. N., Nørgaard Schmidt, S., \& Mayer, P. (2018). Headspace passive dosing of volatile hydrophobic chemicals - Aquatic toxicity testing exactly at the saturation level. Chemosphere, 211, 694-700.

https://doi.org/10.1016/j.chemosphere.2018.07.150

\section{General rights}

Copyright and moral rights for the publications made accessible in the public portal are retained by the authors and/or other copyright owners and it is a condition of accessing publications that users recognise and abide by the legal requirements associated with these rights.

- Users may download and print one copy of any publication from the public portal for the purpose of private study or research.

- You may not further distribute the material or use it for any profit-making activity or commercial gain

- You may freely distribute the URL identifying the publication in the public portal 


\section{Accepted Manuscript}

Headspace passive dosing of volatile hydrophobic chemicals - Aquatic toxicity testing exactly at the saturation level

Lam Ngoc Trac, Stine Nørgaard Schmidt, Philipp Mayer

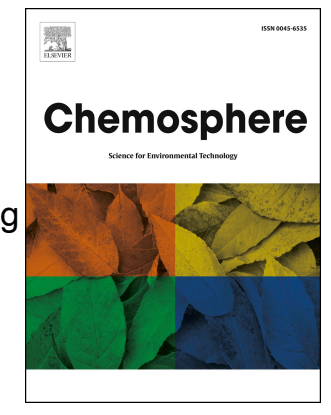

PII:

S0045-6535(18)31415-2

DOI:

10.1016/j.chemosphere.2018.07.150

Reference: CHEM 21865

To appear in: $E C S N$

Received Date: 21 March 2018

Revised Date: 23 July 2018

Accepted Date: 24 July 2018

Please cite this article as: Trac, L.N., Schmidt, Stine.Nø., Mayer, P., Headspace passive dosing of volatile hydrophobic chemicals - Aquatic toxicity testing exactly at the saturation level, Chemosphere (2018), doi: 10.1016/j.chemosphere.2018.07.150.

This is a PDF file of an unedited manuscript that has been accepted for publication. As a service to our customers we are providing this early version of the manuscript. The manuscript will undergo copyediting, typesetting, and review of the resulting proof before it is published in its final form. Please note that during the production process errors may be discovered which could affect the content, and all legal disclaimers that apply to the journal pertain. 


\section{Authors:}

5 Lam Ngoc Trac*, Stine Nørgaard Schmidt, Philipp Mayer

6 Address:

7 Department of Environmental Engineering, Technical University of Denmark, DK-2800 Kgs. Lyngby,

8 Denmark

9 * Corresponding author: Department of Environmental Engineering, Technical University of Denmark, 10 DK-2800 Kgs. Lyngby, Denmark. Email address: nltr@env.dtu.dk 
13

14

15

\section{Abstract}

It is challenging to conduct aquatic tests with highly hydrophobic and volatile chemicals while avoiding substantial sorptive and evaporative losses. A simple and versatile headspace passive dosing (HS-PD) method was thus developed for such chemicals: The pure liquid test chemical was added to a glass insert, which was then placed with the open end in the headspace of a closed test system containing aqueous test medium. The test chemical served as the dominating partitioning donor for establishing and maintaining maximum exposure levels in the headspace and aqueous solution, without direct contact between the donor and the test medium. The HS-PD method was cross validated against passive dosing with a saturated silicone elastomer, using headspace gas chromatography as analytical instrument and saturated vapors as reference. The HS-PD method was then applied to control the exposure in algal growth inhibition tests with the green algae Raphidocelis subcapitata. The model chemicals were C9-C14 n-alkanes and the cyclic volatile methyl siloxanes octamethyltetracyclosiloxane (D4) and decamethylpentacyclosiloxane (D5). Growth rate inhibition at the solubility limit was $100 \%$ for C9-C13 n-alkanes and $53 \pm 31 \%$ (95\% Cl) for tetradecane. A moderate inhibition of $11 \pm 4 \%(95 \% \mathrm{Cl}$ ) was observed for D4, whereas no inhibition was observed for D5. The present study introduces an effective method for aquatic toxicity testing of a difficultto-test group of chemicals and provides an improved experimental basis for investigating toxicity cut-offs.

\section{Keywords: algal growth inhibition, solubility limit, high Henry's constants, n-alkanes, siloxanes, toxicity} cut-offs. 


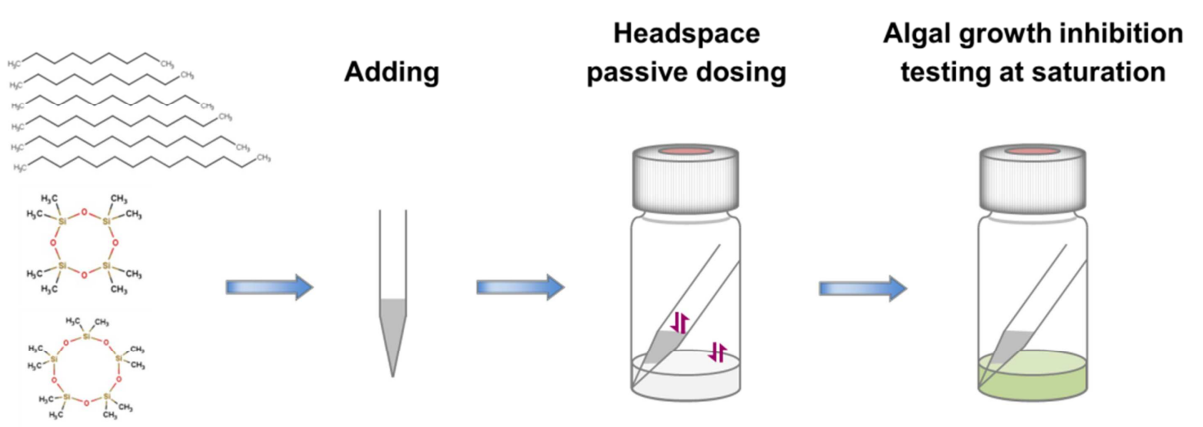

33

- Passive dosing via the headspace established and maintained constant exposure.

- Headspace passive dosing was cross validated by silicone rod passive dosing.

- Aquatic toxicity testing of volatile hydrophobic chemicals exactly at saturation.

39

- Algal growth inhibition limit tests showed toxicity of alkanes and D4, but not D5.

40

41 


\section{Introduction}

The environmental risk assessment of organic chemicals requires proper aquatic toxicity testing, which for some chemicals is straightforward, whereas it for other chemicals is associated with substantial scientific, technical, and analytical challenges (OECD, 2000; Rufli et al., 1998). The quality of the risk assessment of such difficult-to-test chemicals will then highly depend on whether the available test methodologies are fit for the purpose. There are several physical-chemical properties that can make chemicals difficult to test, including limited stability, light attenuation (color), pH sensitive speciation, and surface active properties (OECD, 2000; Rufli et al., 1998). However, two of the most often encountered properties that lead to experimental difficulties are high hydrophobicity and high volatility. High hydrophobicity is generally indicated at octanol to water partition coefficients $\left(\mathrm{K}_{\text {ow }}\right)$ above $10^{5}$ (i.e., $\log \mathrm{K}_{\text {ow }}>5$ ) and at aqueous solubility in the ng- $\mu \mathrm{g}$ per liter range. Highly hydrophobic chemicals can be difficult (1) to introduce into the test medium, (2) to keep at constant concentrations during the test, and (3) to test up to the solubility limit while avoiding the presence of pure chemical in the test medium. High volatility is in aquatic toxicity testing best indicated by high air to water partition coefficients ( $\mathrm{K}_{\text {air,water }}$ ) (unit less Henry's constant, $L L^{-1}$ ). Chemicals with $\mathrm{K}_{\text {air,water }}>1$ are particularly difficult to test since they are prone to substantial evaporative losses, even when tested in closed test systems, due to their high tendency to partition into the headspace (Birch et al., 2017; Mayer et al., 2000). There are about 3900 chemicals with $K_{\text {air,water }}>1$ and Log $K_{\text {ow }}>5$ registered in the Danish QSAR database (Danish (Q)SAR Database, 2018), which are difficult to test with currently available methods.

During the last two decades, a range of passive dosing techniques have been developed and applied for controlling the exposure to hydrophobic organic chemicals (HOCs) in various aquatic toxicity tests (Bragin et al., 2016; Butler et al., 2013; Mayer et al., 1999; Seiler et al., 2014; Smith et al., 2010a, 2010b; Stibany et al., 2017). In passive dosing, a partitioning donor is loaded with the test chemical(s) and applied to establish well-defined concentrations at the start of the test, and then to maintain constant concentrations during 
the test (Smith et al., 2010a). While passive dosing has been successfully applied in many studies, there are still several challenges and a need for further developments: (1) Passive dosing has mainly been applied to solid HOCs (Mayer and Holmstrup, 2008; Smith et al., 2013, 2010a) and to liquid HOCs with moderate volatility (Stibany et al., 2017), and it is thus crucial to extend this work to liquid HOCs with high volatility.

(2) Many passive dosing formats require substantial preparative work prior to the toxicity tests (Smith et al., 2010a), and time efficient passive dosing methods suited for high throughput testing are urgently needed (Vergauwen et al., 2015).

Recently, a new passive dosing method was developed for testing liquid HOCs exactly at the solubility limit (i.e., limit testing) (Stibany et al., 2017). This method employed a silicone O-ring, which was first loaded to saturation by direct immersion in the pure liquid test chemical. The saturated silicone was then placed in the test medium for controlling the exposure exactly at the saturation level. This approach is simple, practical, and leads also to reproducible toxicity data. Although not observed in the specific study by Stibany et al. (2017), direct contact between a saturated donor and test medium could, in worst case, lead to micro droplets entering the test medium, which would then compromise the interpretation of the observed effects. This calls for novel passive dosing methods that exclude the direct contact between the passive dosing donor and the test medium.

The present study introduces a new, simple, and versatile passive dosing method for aquatic toxicity testing of volatile HOCs (i.e., liquid chemicals with high Henry's constants), with no direct contact between the donor and the test medium. This new passive dosing approach is operated via the headspace (i.e., headspace passive dosing, HS-PD): The test chemical itself acts as the donor that quickly saturates the headspace through vaporization, which then saturates the aqueous test medium by air water equilibrium partitioning. The donor is easily administrated by adding the pure test chemical to an insert, which is then placed directly in the closed test vial containing aqueous test medium and headspace (see the graphical abstract for visualization of the system). Through the vapor phase, the test chemical is introduced into the 
test medium as individual molecules, thus avoiding the introduction of micro droplets. However, re-condensation of test chemical on the inner surfaces of the test system cannot be ruled out when operating the method exactly at the saturation level. Testing just below the saturation limit should therefore be included to elaborate this condensation effect.

The careful control and measurement of exposure in environmental testing have implications well beyond the technical level. In fact, several passive dosing studies have already shown how improved exposure control can lead to fundamental new results and also provide a better understanding of toxicity (Schmidt et al., 2013; Seiler et al., 2014; Stibany et al., 2017), biodegradation (Birch et al., 2018, 2017; Smith et al., 2012), toxicity cut-offs (Mayer and Holmstrup, 2008), and mixture toxicity (Bjergager et al., 2017; Smith et al., 2013). On the scientific level, the present study aimed to determine the aquatic toxicity of $n$-alkanes and cyclic volatile methyl siloxanes (cVMS). N-alkanes are significant constituents of petroleum products (De Graaff et al., 2012) and used as intermediates in the production of surfactants and detergents (American Chemistry Council n-Alkane VCCEP Consortium, 2004). The $n$-alkanes with the number of carbon atoms ranging from 9-14 (C9-C14) were selected as model chemicals because they have a high Henry's constant, their toxicity to aquatic organisms is still in question, and they comprise a wide range of carbon chain length, which makes them suitable for studying cut-offs in toxicity. The cVMS D4 (octamethylcyclotetrasiloxane) and D5 (decamethylcyclopentasiloxane) were also selected, since they are widely used in personal care products as well as industrial applications (Fairbrother et al., 2015; Fairbrother and Woodburn, 2016). They are chemicals of emerging concern and have become the subject of interest for both scientific and regulatory scrutiny, since they have been detected and monitored in air, water, sediments, and biota (Gobas et al., 2015; Mackay et al., 2015a). Their high volatility and low solubility present challenges for assessing the ecological risks of these chemicals to aquatic systems (Fairbrother and Woodburn, 2016; Mackay et al., 2015b). 
113 Therefore, the aim of the present study is twofold: (1) to develop and validate a simple and versatile 114 headspace passive dosing method for testing liquid chemicals with high Henry's constants, which are 115 difficult to test with currently available methods, and (2) to apply the method to the green algae Raphidocelis subcapitata (formerly known as Pseudokirchneriella subcapitata and Selenastrum capricornutum) growth inhibition tests with n-alkanes and siloxanes at, and just below, their saturation levels to investigate the condensation effects and better understand their aquatic toxicity. The hypotheses of the study are: (1) The headspace passive dosing approach can provide exposure exactly at the saturation limit by equilibrium partitioning between the liquid test chemical and the aqueous test medium via the headspace, and (2) n-alkanes C9-C14 and siloxanes D4 and D5 can exert toxicity to the algae $R$. subcapitata when tested at the maximum exposure level, i.e., at chemical activity of unity (Reichenberg and Mayer, 2006).

\section{Materials and methods}

Clear 20-mL glass vials (ND18, $75.5 \times 22.5 \mathrm{~mm}$ and ND24, $57 \times 27.5 \mathrm{~mm}$ ), screw caps with Teflon-lined (PTFE) septa, and 0.3-mL glass inserts were purchased from Mikrolab Aarhus A/S, Denmark. Pyrex ${ }^{\circledR}$ fiber glass wool ( $8 \mu \mathrm{m}$ per strand) was from Sigma-Aldrich (Denmark). Translucent silicone rods with a diameter of $3 \mathrm{~mm}$ were custom made by Altec Products Ltd (United Kingdom) and used as partitioning donors in the silicone rod passive dosing system (see Section 2.3.). The test chemicals were nonane ( $\geq 99 \%$, Fluka AG, Buchs SG, Switzerland), decane ( $\geq 99 \%$, Sigma-Aldrich, Denmark), undecane ( $\geq 99 \%$, Sigma-Aldrich Chemie, Germany), dodecane (>99\%, TCl Chemicals, Belgium), tridecane ( $\ 99 \%$, Fluka AG, Switzerland), tetradecane ( $\geq 99 \%$, Fluka AG, Switzerland), octamethylcyclotetrasiloxane (D4, 98\%, Sigma-Aldrich, Denmark), and decamethylcyclopentasiloxane (D5, 97\%, Sigma-Aldrich, Denmark). Miglyol 812 oil was used for diluting the pure test chemicals and was from Cremer Oleo GmbH \& Co. KG, Germany. Ethanol and acetone were purchased from VWR (HPLC grade, VWR International GmbH, Germany). Milli-Q water (LaboStar ${ }^{\mathrm{TM}}$ 1-DI 
ultra-pure water system, Evoqua water technologies, Germany) was used in algal test medium and for washing the silicone rods. Lint-free tissues (Assistent, Germany) were used for drying the silicone rods. Isotone (Coulter isotone II diluent, Beckman Coulter GmbH, Germany) was used as diluent for coulter counting to determine algal cell densities in the pre-cultures.

\subsection{Development of the headspace passive dosing method}

The headspace passive dosing (HS-PD) approach was developed to enable testing of chemicals with high $\mathrm{K}_{\text {ow }}$ and high $\mathrm{K}_{\text {air,water, }}$ while avoiding the introduction of pure phase test chemical into the test medium and minimizing the preparatory work prior to toxicity testing. Specifically, $0.1 \mathrm{~mL}$ of a liquid test chemical was simply added to a $0.3-\mathrm{mL}$ glass insert, which was then placed in a 20-mL ND24 glass vial (test vial) containing $4 \mathrm{~mL}$ of algal test medium (see the graphical abstract for visualization of the system). The pure test chemical thus acted as the dominating partitioning donor controlling the exposure in the headspace as well as in the aqueous solution by equilibrium partitioning. Glass wool was placed in the inserts to increase the surface area and thereby facilitate the partitioning process between the pure phase and the headspace. The test vials were sealed tight, and the complete system was then shaken ( $200 \mathrm{rpm}, 10 \mathrm{~mm}$ orbit) for $24 \mathrm{~h}$ at $20 \pm 1{ }^{\circ} \mathrm{C}$ to ensure equilibrium between the donor, headspace, and test medium at test start (preequilibration). The pre-equilibration was performed in the dark to avoid photodecomposition.

Exposure of a given test chemical at its saturation level in the HS-PD system was confirmed by headspace gas chromatography with flame ionization detector (headspace GC-FID) using saturated vapor over the pure liquid as reference. The saturated vapor of a test chemical was simply prepared by adding $1 \mathrm{~mL}$ of the chemical into a test vial and subsequently shaking the vial (200 rpm, $10 \mathrm{~mm}$ orbit) for $24 \mathrm{~h}$ at $20 \pm 1{ }^{\circ} \mathrm{C}$ in the dark.

2.3. Cross validation by the silicone rod passive dosing method 
Passive dosing using a saturated silicone polymer is a well-established and reproducible approach for controlling the exposure in aquatic toxicity testing of hydrophobic organic chemicals (HOCs) exactly at the saturation limit (Smith et al., 2010a; Stibany et al., 2017). This approach was therefore used to cross validate the HS-PD approach. For preparing the silicone donors, a long silicone rod was first soaked in excess ethanol and shaken overnight to remove dust and impurities. The rod was then rinsed three times with excess Milli-Q water during a period of $>24 \mathrm{~h}$ to remove ethanol and dried with lint-free tissues. Then, the rod was cut into pieces of approximately $4 \mathrm{~cm}$ and weighed. The loading was done in closed 20-mL ND18 glass vials, where the silicone rods were immersed in excess pure test chemical for at least $24 \mathrm{~h}$.

Preliminary experiments showed that $24 \mathrm{~h}$ is sufficient for the test chemicals to reach saturation in silicone rods, indicated by no additional weight gain in the loaded rods (results not shown). The resulting saturated silicone rods were rinsed three times with Milli-Q water, dried with lint-free tissues, re-weighed, and eventually used for establishing and controlling the exposure in the test system. They were placed standing upright in the test vials containing $4 \mathrm{~mL}$ of algal test medium (see Figure S1 for visualization of the system). In this way, the exposure in the aqueous medium was controlled by equilibrium partitioning via the headspace and by direct passive dosing from the silicone rod. The test vials were then shaken $(200 \mathrm{rpm}, 10$ $\mathrm{mm}$ orbit) for $24 \mathrm{~h}$ for pre-equilibration. Both the loading and pre-equilibration were performed at $20 \pm 1{ }^{\circ} \mathrm{C}$ in the dark. The exposure levels in this passive dosing system were also measured using headspace GC-FID and compared to the results from the HS-PD method and the saturated vapors over the pure liquids (see Section 2.2).

\subsection{GC-FID analysis}

Thermo Scientific $^{\text {TM }}$ Trace $^{\text {TM }} 1300$ GC-FID was used for quantifying test chemicals. A 50- $\mu$ L air sample was taken from the headspace of test vials using a Hamilton $50-\mu \mathrm{L}$ gas tight syringe and manually injected. The syringe was cleaned with ethanol vapor and flushed with air for drying between samplings, and none of the test compounds was detected in control injections. The instrument was operated with a Trace ${ }^{\mathrm{TM}} \mathrm{TR}-50.53$ 
$\mathrm{mm}, 1.5 \mu \mathrm{m}, 30 \mathrm{~m}$ column (Thermo Scientific, Italy), an injection temperature of $150{ }^{\circ} \mathrm{C}$ with a split ratio of 3:1 and a helium carrier flow rate of $3 \mathrm{~mL} \mathrm{~min}^{-1}$. The temperature was initially set to $40{ }^{\circ} \mathrm{C}$, held for $2 \mathrm{~min}$, increased by $25^{\circ} \mathrm{C} \mathrm{min}^{-1}$ up to $180^{\circ} \mathrm{C}$ and finally held for $1 \mathrm{~min}$. Peak area integration was accurately performed with Chromeleon ${ }^{\mathrm{TM}} 7.2 \mathrm{CDS}$ software (Thermo Scientific).

\subsection{Mass distribution calculations}

The mass distribution of test chemicals in the two passive dosing systems, i.e., headspace and silicone rod, was estimated to illustrate the large buffer capacity of the donor and the high partitioning of the test chemicals to the headspace compared to the aqueous phase. The test systems consisted of the following compartments: a partitioning donor, a headspace, and an aqueous medium when assuming negligible adsorption to the glass and PTFE materials. The mass of test chemical in the donor phase of the HS-PD system was calculated based on the volume (i.e., $0.1 \mathrm{~mL}$ ) and relative density of a given liquid, and the loaded mass of the silicone rod was calculated as the weight differences before and after loading with test chemicals. The mass of test substance in the headspace $\left(m_{H S}, \mathrm{~g}\right)$ was calculated based on the ideal gas law:

$$
m_{H S}=\left(p \times M \times V_{H S}\right) /(R \times T)
$$

where $p$ is vapor pressure (Pa), $M$ is molar mass $\left(\mathrm{g} \mathrm{mol}^{-1}\right), V_{H S}$ is the headspace volume (estimated to be $15.1 \mathrm{~mL}$, considering $0.9 \mathrm{~mL}$ is taken up by the insert or silicone rod), $R$ is universal gas constant ( $\mathrm{Pa} \mathrm{m}^{3} \mathrm{~mol}^{-}$ $\left.{ }^{1} \mathrm{~K}^{-1}\right)$, and $T$ is absolute temperature (298 K). The water solubility values of the test substances were used for calculating the masses in the test medium. Physical-chemical property data were obtained from Environmental Risk Assessment reports (Brooke et al., 2009a, 2009b) for siloxanes and from Handbook of Physical-Chemical Properties and Environmental Fate for Organic Chemicals (Mackay et al., 2006) for nalkanes.

2.6. Algal growth inhibition tests at the solubility limit 
The Raphidocelis subcapitata growth inhibition test was based on the OECD guideline 201 (OECD, 2011). A small adjustment, i.e., a $150 \mathrm{mg} \mathrm{L}^{-1} \mathrm{NaHCO}_{3}$ enrichment of the algal medium, was made to buffer $\mathrm{pH}$ changes in the closed test system (Mayer et al., 2000). Pre-cultures were maintained for a week before test start with a renewal interval of every other day. The algal cell density was determined using the Coulter ${ }^{\circledR}$ Particle Counter Z1 (Beckman Coulter GmbH, Germany).

First, the HS-PD approach was applied to maintain the exposure to the test chemical exactly at the saturation limit. Pre-equilibration was completed prior to test start to ensure equilibrium of test chemicals among all phases in the test system, as described in Section 2.2. Second, an additional treatment with approximately $80 \%$ pure test chemical was included to investigate the condensation effect in the test system (i.e., the toxic effect that might have been caused by condensed pure test chemical in the system). The donor phase of this treatment was prepared by mixing the pure test chemical with Miglyol oil with a ratio of 4:1 (w:w). Therefore, oil control samples were included and contained $0.1 \mathrm{~mL}$ Miglyol oil only in the inserts. The global control samples, which were used to check the validity of the test as well as a reference to calculate growth inhibition, contained $4 \mathrm{~mL}$ of algal medium only.

All tests were conducted as described by Stibany and colleagues (Stibany et al., 2017). Briefly, the pre-equilibrated test vials were opened (one vial at a time) and algal pre-culture was added to reach an initial algal density in each vial of $10^{4}$ cells mL ${ }^{-1}$. Then, the test vials were sealed and $175 \mu \mathrm{LCO}_{2}$ was added to the headspace (using a syringe) to ensure sufficient supply of $\mathrm{CO}_{2}$ during the closed test (HallingSørensen et al., 1996). Test vials were shaken (300 rpm, $3 \mathrm{~mm}$ orbit) at $20 \pm 1{ }^{\circ} \mathrm{C}$ under continuous illumination from beneath. The light intensity was $123 \pm 3 \mu \mathrm{mol} \mathrm{m} \mathrm{m}^{-2} \mathrm{~s}^{-1}$ photosynthetically active range (PAR) photons. After 24,48 , and $72 \mathrm{~h}$, algal biomass was measured by in vitro chlorophyll fluorescence (Mayer et al., 1997) and used in the calculation of the specific growth rate $\left(\mu, \mathrm{d}^{-1}\right)$ :

$$
\mu=\left(\ln \left(N_{t}\right)-\ln \left(N_{0}\right)\right) / \mathrm{t}
$$


where $N_{t}$ is the algal biomass at sampling time $t(d)$ and $N_{0}$ is algal biomass at test start. The toxic effect was calculated as the percent reduction of growth rate $(I, \%)$ relative to the control (without insert) for each treatment replicate:

$$
I=\left(1-\mu_{\text {treatment }} / \mu_{\text {control }}\right) \times 100
$$

In accordance with the ISO 8692 guideline (DIN, 2012), a minimum growth rate of 1.4 day $^{-1}$ in the controls was used as validity criterion for the tests.

For n-alkanes, the algal growth inhibition test was performed once with six control replicates and three sample replicates for each chemical. For D4 and D5, three separate tests were performed with the same replicate setup (except the first test with just three control replicates). The validity criterion was not met in the third test with the siloxanes, and the average results of the first and second tests were therefore used.

\subsection{Data treatment}

One-way analysis of variance (ANOVA) was conducted to test significant differences in exposure levels between the HS-PD system, the silicone rod system, and the saturated vapors. Pairwise multiple comparisons were performed following the one-way ANOVA test using Holm-Sidak's method in case there were differences. Dunnett's t-test was conducted to test significant differences in algal growth rates between treatments and global controls. Data were checked for normal distribution and homogeneity of variances by Kolmogorov-Smirnov's test and Levene's test, respectively. All statistical analyses were performed using SigmaPlot 11.0 (Systat Software Inc., California, USA) with a significance level of 0.05 (i.e., $p<0.05)$. For the presentation of algal growth inhibition caused by $n$-alkanes, data from treatments that showed more than $100 \%$ inhibition were set to $100 \%$ and then used for analysis.

\section{Results and discussion}

3.1. Headspace passive dosing and cross validation by silicone rod passive dosing 
249 After $24 \mathrm{~h}$ of equilibration, measurements of $\mathrm{n}$-alkanes and siloxanes in the headspace of both passive 250 dosing systems corresponded well to the reference measurements of the saturated vapors over the pure 251 liquids (Figure 1). There were some minor but statistically significant differences, which we attribute to 252 technical issues and particularly a minor temperature difference between the equilibration period and 253 waiting time prior to manual injection for GC-FID analysis. For most test substances, there was no 254 statistically significant difference in the measured exposure levels between the two passive dosing systems. 255 The only real exception was C9 with measurements in the silicone rod system being lower compared to the 256 measurements in the HS-PD system as well as to the reference system (Figure 1A). Differences in exposure 257 in cases of C10 and C11 were statistically significant between the two passive dosing methods and the 258 reference system, but less than $30 \%$. Overall, the analytical results confirmed that both passive dosing 259 systems were able to establish exposure of the test chemicals at their saturation level within $24 \mathrm{~h}$ 260 equilibration. Furthermore, results from the two passive dosing methods cross validated each other based 261 on the good agreement between measurements in the headspace. This makes the two methods 262 compatible and well aligned. 

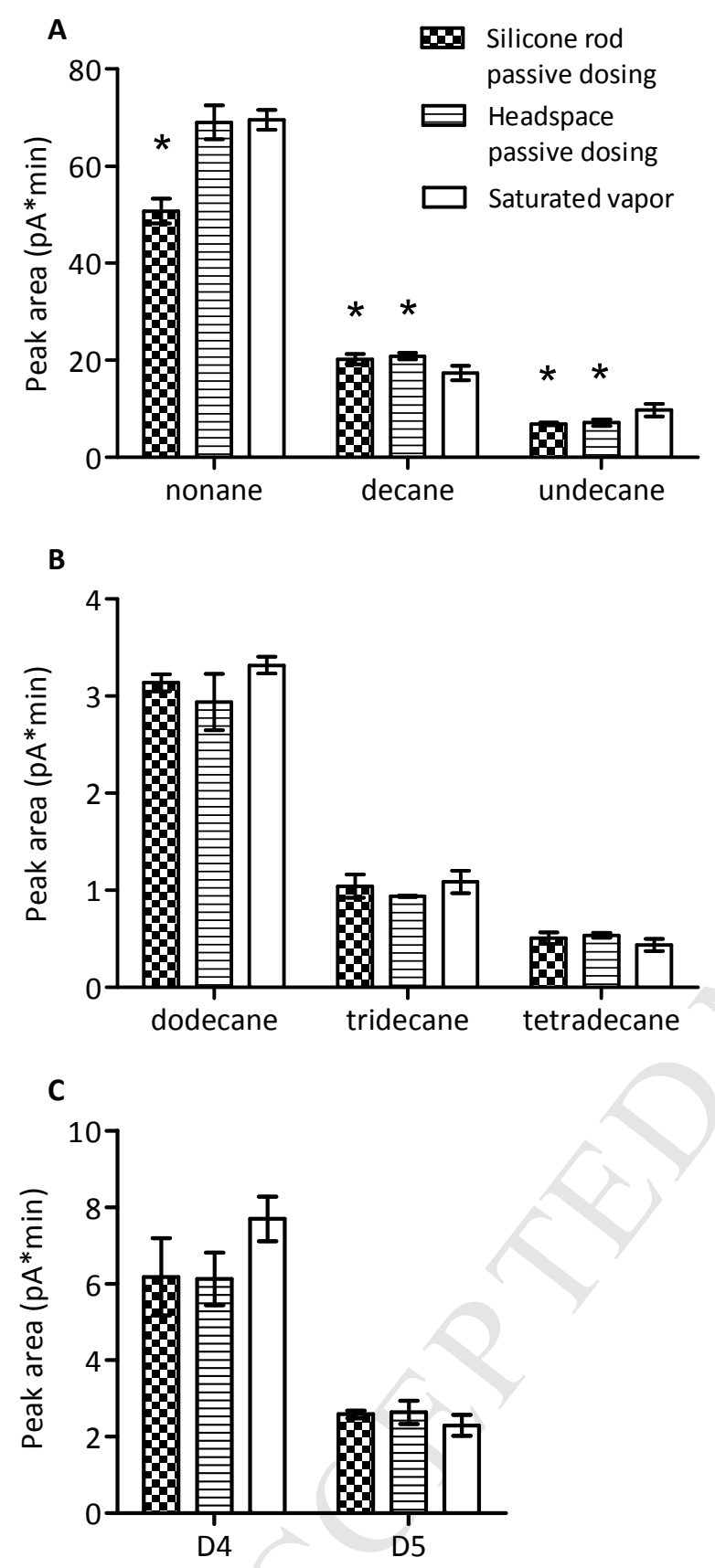

264 Figure 1: Exposure levels of $n$-alkanes ( $A$ and $B$ ) and siloxanes $(C)$ in two passive dosing systems (silicone rod 265 and headspace, respectively) and their saturated vapors after $24 \mathrm{~h}$ of equilibration (mean $\pm S D, n=3$ ).

266 Asterisks indicate statistically significant differences from corresponding saturated vapors $(p<0.05)$. 
267 It has been shown in previous studies that mass transfer of volatile hydrophobic organic chemicals (HOCs)

268 between water and headspace in 2-20 mL closed test systems is fast (Mayer et al., 2000; Zhang and

269 Pawliszyn, 1993). Indeed, this is the reason for the very large and fast evaporative losses of volatile organic

270 test substances in, for instances, algal growth inhibition testing (Mayer et al., 2000), and the basis for the

271 analytical technique Headspace Solid-Phase Microextraction (HS-SPME) (Zhang and Pawliszyn, 1993). In our

272 HS-PD system, fast mass transfer of test chemical into the aqueous medium takes place via the headspace

273 and through vaporization of test chemical from the donor. At equilibrium, aqueous concentrations are at

274 maximum solubility when headspace concentrations are at the saturation limit. Therefore, measuring test

275 chemicals in the headspace of the two passive dosing systems and relating these results to saturated vapor

276 references was a fast and precise approach to confirm the exposure at saturation.

\section{3.2. Mass distribution}

The mass distribution calculations illustrate that chemicals with high Henry's constants distribute more to the headspace than to the aqueous phase (Table 1). Specifically, the amount of $n$-alkane was about 70 to 3400 times (for nonane and undecane, respectively) higher in the headspace than in the test medium, while the differences were about 1100 times for the siloxanes. This implies very high evaporative losses of such chemicals to the headspace, and thus a dramatic decrease of aqueous medium concentrations when applying methods not optimized for chemicals with high Henry's constants (Birch et al., 2017; Mayer et al., 2000). The mass distribution calculation also emphasizes that the passive dosing donor, at equilibrium, carries at least $99.4 \%$ (for nonane) of the test chemical in the test system, which means that the donor phase is truly the dominating partitioning source for controlling the concentration in the test medium. This buffer capacity is highly sufficient to replenish the aqueous medium with test chemicals in case of losses and can therefore maintain constant exposure of the test chemicals throughout the 72-h test period. 
290 Table 1: Mass distribution of $n$-alkanes and cyclic volatile methyl siloxanes in the silicone rod (Rod) and 291 headspace (HS) passive dosing systems.

\begin{tabular}{|c|c|c|c|c|c|c|}
\hline & Rod & $\mathrm{HS}$ & Rod & $\mathrm{HS}$ & Rod & $\mathrm{HS}$ \\
\hline & \multicolumn{2}{|c|}{ nonane } & \multicolumn{2}{|c|}{ decane } & \multicolumn{2}{|c|}{ Undecane } \\
\hline Donor (\%) & 99.87 & 99.35 & 99.95 & 99.78 & 99.98 & 99.93 \\
\hline Headspace (\%) & 0.13 & 0.64 & 0.048 & 0.22 & 0.018 & 0.074 \\
\hline \multirow[t]{2}{*}{ Medium (\%) } & $1.9 e-03$ & $9.5 e-03$ & $1.8 \mathrm{e}-05$ & $8.3 e-05$ & $5.3 e-06$ & $2.2 \mathrm{e}-05$ \\
\hline & \multicolumn{2}{|c|}{ dodecane } & \multicolumn{2}{|c|}{ tridecane } & \multicolumn{2}{|c|}{ Tetradecane } \\
\hline Donor (\%) & 99.99 & 99.97 & 99.997 & 99.99 & 99.999 & 99.997 \\
\hline Headspace (\%) & 7.0e-03 & 0.025 & 2.7e-03 & $8.5 \mathrm{e}-03$ & $1.1 \mathrm{e}-03$ & $2.9 \mathrm{e}-03$ \\
\hline Medium (\%) & $5.5 e-06$ & 2.0e-05 & $1.7 \mathrm{e}-06$ & $5.5 e-06$ & $6.7 e-07$ & $1.7 \mathrm{e}-06$ \\
\hline \multicolumn{7}{|c|}{ D4 } \\
\hline Donor (\%) & 99.95 & 99.75 & 99.98 & 99.92 & & \\
\hline Headspace (\%) & 0.048 & 0.25 & 0.016 & 0.079 & & \\
\hline Medium (\%) & $4.4 \mathrm{e}-05$ & $2.3 e-04$ & $1.4 \mathrm{e}-05$ & 7.1e-05 & & \\
\hline
\end{tabular}

294 When tested at the saturation level, all n-alkanes caused full inhibition of the algal growth rate, except for 295 tetradecane, causing $53 \pm 31 \%(95 \% \mathrm{Cl}$ ) inhibition (Figure 2). Similarly, full algal growth rate inhibition by 296 C9-C13 was also observed when the test chemicals were tested at $80 \%$ of the saturation level. The toxic 297 effect observed at the $80 \%$ saturation level thus confirmed that the algal growth inhibition was caused by 298 the freely dissolved molecules and not by potential artifacts of condensed micro droplets of the test 299 chemicals in the test system. The lower inhibition by tetradecane indicated a cut-off point in toxicity for 
300 301 $(95 \% \mathrm{Cl})$, whereas D5 did not inhibit the algal growth (Figure 2).

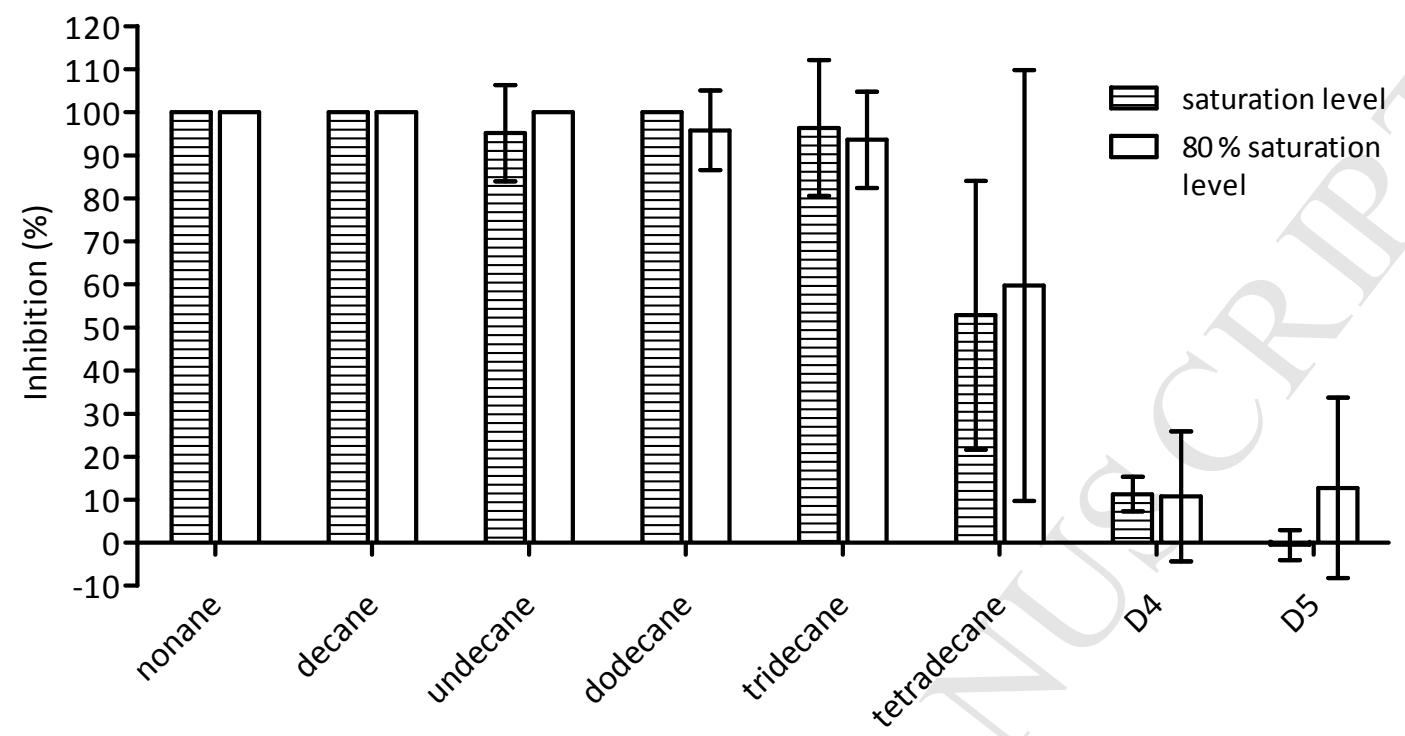

alkanes with longer chain length. For siloxanes, D4 caused moderate growth rate inhibition, i.e., $11 \pm 4 \%$
302

Figure 2: Algal growth inhibition after $72 \mathrm{~h}$ caused by $\mathrm{n}$-alkanes (mean $\pm 95 \% \mathrm{Cl}, \mathrm{n}=3$ replicates) and siloxanes (mean $\pm 95 \% \mathrm{Cl}, \mathrm{n}=6$ replicates) at the saturation and $80 \%$ saturation levels. Inhibition results from two individual tests for the siloxanes are shown in the supporting information (Figures S2 and S3).

D4 and D5 were less toxic than expected (second hypothesis), since they exerted no or only limited toxicity even when tested at the solubility limit (i.e., chemical activity of unity). To the contrary, tetradecane exerted approximately 50\% growth inhibition at the solubility limit, despite its Log $\mathrm{K}_{\text {ow }}$ of 8.0 (Mackay et al., 2006) being well above the reported hydrophobicity cut-off for baseline toxicity at Log $K_{\text {ow }}$ of 6 (Könemann, 1981). A similar finding was recently observed for dodecylbenzene (DDB) with a Log $K_{\text {ow }}$ of 8.65 , which was found to be moderately toxic towards algae when tested at the solubility limit (Stibany et al., 2017). These observations are in good agreement with previous studies on the baseline toxicity of highly HOCs (Mayer and Reichenberg, 2006) and on applying bioconcentration kinetics for the prioritization of aquatic toxicity testing of highly HOCs (Kwon et al., 2016). 
The toxicity of D4 and D5 to aquatic organisms is of particular interest for regulatory scrutiny. Regulatory assessment of these chemicals has been conducted by many authorities, including United Kingdom Environment Agency (Brooke et al., 2009b, 2009a), Environment Canada and Health Canada (Environment Canada and Health Canada, 2008a, 2008b), and Danish Environmental Protection Agency (Danish EPA, 2014). The assessment of D5 has been controversial, as D5 was initially considered to be toxic but was later reported not to pose a danger to the environment (Mackay et al., 2015a). Within the regulatory framework, the observations of the present study suggest that D5 had neither acute nor chronic effects on algal growth at the maximum exposure level. D4 exerted a growth rate inhibition of $11 \%$ when tested at the saturation level, which is very similar to the recently tested dodecylbenzene and can be interpreted as a moderate chronic toxicity to algae (Stibany et al., 2017).

From the physical-chemical point of view, the present study demonstrates a simple "plug-and-play" approach to test liquid HOCs with high Henry's constants. High hydrophobicity and volatility are two of the physical-chemical properties that make a chemical difficult to test (OECD, 2000). However, these properties were utilized in the HS-PD approach to enable simple, practical, and also more efficient toxicity testing of such difficult-to-test chemicals. In addition, the introduction of dissolved molecules via the headspace helps avoid the formation of pure chemicals in the test medium. The preparative work for the partitioning donor was also vastly reduced compared to other passive dosing approaches. For instance, in the passive dosing approaches using silicone polymer there are several steps for preparing the donor, which involve: (1) the selection and dimensioning of the donor material to ensure negligible depletion, (2) the careful cleaning of the donor material prior to loading, and (3) the loading of test substances into the donor material (Butler et al., 2013; Engraff et al., 2011; Schmidt et al., 2013; Seiler et al., 2014; Smith et al., 2013). Hence, the simple preparation of the donor also made the HS-PD approach more versatile by allowing testing both at, and just below, the saturation level. This was easily done without any significant effort by diluting the pure chemicals with Miglyol oil to obtain the $80 \%$ saturation level. 
Furthermore, this approach can be applied to other species including fish and invertebrates. However, one should then ensure that (1) the closed test vessel does not lead to significant changes in test conditions (i.e., $\mathrm{pH}$, oxygen content, light intensity, etc.) and also comply with the respective test guidelines, (2) factors that help achieve quick equilibration of test chemicals in the test system should be considered, for example, shaking of test vial and/or increasing surface areas of both test medium and pure test chemical, and (3) the amount of the pure test chemical should be sufficient compared to the amount in the test medium to ensure non-depleted source of test chemical for passive dosing. Further work is thus needed to adapt, optimize, and validate the HS-PD approach for the toxicity testing with other species.

Volatile chemicals can evaporate before and after entering natural aquatic environments, and one might thus ask whether aquatic toxicity testing of these chemicals is necessary. Volatile HOCs are not only prone to evaporation but they also bind to dissolved organic matter and sorb to sludge and sediment. These binding and sorption interactions can then keep volatile HOCs within the aquatic environment for a longer period of time (Panagopoulos and MacLeod, 2018), which poses potential risks to the organisms living in this compartment. It is therefore essential to have the possibility for assessing the environmental impact of such difficult-to-test chemicals and suitable ecotoxicological methods are strongly needed. The two passive dosing methods allow "limit testing at saturation" within a large applicability domain with regards to hydrophobicity and volatility. These approaches can now be used for simplifying and improving the regulatory toxicity testing of volatile hydrophobic organic chemicals, and also for more dedicated studies exploring toxicity cut-off phenomena and challenging existing toxicity cut-off theories.

\section{Acknowledgement}

The authors thank Hanne Bøggild and Mikael Emil Olsson for technical and analytical support. This paper is part of a Ph.D. study funded by the Technical University of Denmark. We acknowledge Unilever, Shell, and the National Centre for the Replacement, Refinement and Reduction of Animals in Research (NC3Rs) for additional funding (Crack-it Challenge 27: DoCE). 


\section{References}

American Chemistry Council n-Alkane VCCEP Consortium, 2004. N-Alkane Category: decane, undecane, dodecane. Voluntary Children's Chemical Evaluation Program (VCCEP) submission.

Birch, H., Andersen, H.R., Comber, M., Mayer, P., 2017. Biodegradation testing of chemicals with high Henry's constants - Separating mass and effective concentration reveals higher rate constants. Chemosphere 174, 716-721. doi:10.1016/j.chemosphere.2017.02.003

Birch, H., Hammershøj, R., Mayer, P., 2018. Determining biodegradation kinetics of hydrocarbons at low concentrations: Covering 5 and 9 orders of magnitude of Kow and Kaw. Environ. Sci. Technol. 52, 2143-2151. doi:10.1021/acs.est.7b05624

Bjergager, M.B.A., Dalhoff, K., Kretschmann, A., Nørgaard, K.B., Mayer, P., Cedergreen, N., 2017. Determining lower threshold concentrations for synergistic effects. Aquat. Toxicol. 182, 79-90. doi:10.1016/j.aquatox.2016.10.020

Bragin, G.E., Parkerton, T.F., Redman, A.D., Letinksi, D.J., Butler, J.D., Paumen, M.L., Sutherland, C.A., Knarr, T.M., Comber, M., den Haan, K., 2016. Chronic toxicity of selected polycyclic aromatic hydrocarbons to algae and crustaceans using passive dosing. Environ. Toxicol. Chem. 35, 2948-2957. doi:10.1002/etc.3479

Brooke, D.N., Crookes, M.J., Gray, D., Robertson, S., 2009a. Environmental Risk Assessment Report: Octamethylcyclotetrasiloxane, UK Environment Agency. Bristol, UK.

Brooke, D.N., Crookes, M.J., Gray, D., Robertson, S., 2009b. Environmental Risk Assessment Report: Decamethylcyclopentasiloxane, UK Environment Agency. Bristol, UK.

Butler, J.D., Parkerton, T.F., Letinski, D.J., Bragin, G.E., Lampi, M.A., Cooper, K.R., 2013. A novel passive 
dosing system for determining the toxicity of phenanthrene to early life stages of zebrafish. Sci. Total Environ. 463-464, 952-958. doi:10.1016/j.scitotenv.2013.06.079

Danish (Q)SAR Database, 2018. URL http://qsar.food.dtu.dk/ (accessed 7.10.18).

Danish EPA, 2014. Siloxanes (D3, D4, D5, D6, HMDS).

De Graaff, R., Forbes, S., Gennart, J.P., Gimeno Cortes, M.J., Hovius, H., King, D., Kleise, H., Martinez Martin, REACH - Analytical characterisation of petroleum UVCB substances, CONCAWE Report no. 7/12.

DIN, 2012. EN ISO 8692: Water quality - Fresh water algal growth inhibition test with unicellular green algae.

Engraff, M., Solere, C., Smith, K.E.C., Mayer, P., Dahllöf, I., 2011. Aquatic toxicity of PAHs and PAH mixtures at saturation to benthic amphipods: Linking toxic effects to chemical activity. Aquat. Toxicol. 102, 142-149. doi:10.1016/j.aquatox.2011.01.009

Environment Canada, Health Canada, 2008a. Screening Assessment for the Challenge Octamethylcyclotetrasiloxane (D4).

Environment Canada, Health Canada, 2008b. Screening Assessment for the Challenge Decamethylcyclopentasiloxane (D5).

Fairbrother, A., Burton, G.A., Klaine, S.J., Powell, D.E., Staples, C.A., Mihaich, E.M., Woodburn, K.B., Gobas, F.A.P.C., 2015. Characterization of ecological risks from environmental releases of decamethylcyclopentasiloxane (D5). Environ. Toxicol. Chem. 34, 2715-2722. doi:10.1002/etc.3041

Fairbrother, A., Woodburn, K.B., 2016. Assessing the Aquatic Risks of the Cyclic Volatile Methyl Siloxane D4. Environ. Sci. Technol. Lett. 3, 359-363. doi:10.1021/acs.estlett.6b00341 
405

406

407

408

409

410

411

412

413

414

415

416

417

418

419

420

421

422

423

424

425

426

Gobas, F.A.P.C., Xu, S., Kozerski, G., Powell, D.E., Woodburn, K.B., Mackay, D., Fairbrother, A., 2015.

Fugacity and activity analysis of the bioaccumulation and environmental risks of decamethylcyclopentasiloxane (D5). Environ. Toxicol. Chem. 34, 2723-2731. doi:10.1002/etc.2942

Halling-Sørensen, B., Nyholm, N., Baun, A., 1996. Algal toxicity tests with volatile and hazardous compounds in air-tight test flasks with CO2 enriched headspace. Chemosphere 32, 1513-1526. doi:10.1016/00456535(96)00059-8

Könemann, H., 1981. Quantitative structure-activity relationships in fish toxicity studies Part 1: Relationship for 50 industrial pollutants. Toxicology 19, 209-221. doi:10.1016/0300-483X(81)90130-X

Kwon, J.-H., Lee, S.-Y., Kang, H.-J., Mayer, P., Escher, B.I., 2016. Including Bioconcentration Kinetics for the Prioritization and Interpretation of Regulatory Aquatic Toxicity Tests of Highly Hydrophobic Chemicals. Environ. Sci. Technol. 50, 12004-12011. doi:10.1021/acs.est.6b03942

Mackay, D., Cowan-Ellsberry, C.E., Powell, D.E., Woodburn, K.B., Xu, S., Kozerski, G.E., Kim, J., 2015a. Decamethylcyclopentasiloxane (D5) environmental sources, fate, transport, and routes of exposure. Environ. Toxicol. Chem. 34, 2689-2702. doi:10.1002/etc.2941

Mackay, D., Powell, D.E., Woodburn, K.B., 2015b. Bioconcentration and Aquatic Toxicity of Superhydrophobic Chemicals: A Modeling Case Study of Cyclic Volatile Methyl Siloxanes. Environ. Sci. Technol. 49, 11913-11922. doi:10.1021/acs.est.5b03195

Mackay, D., Shiu, W.Y., Ma, K., Lee, S.C., 2006. Handbook of Physical-Chemical Properties and Environmental Fate for Organic Chemicals, Second. ed. CRC/Taylor \& Francis. doi:10.1201/9781420044393

Mayer, P., Cuhel, R., Nyholm, N., 1997. A simple in vitro fluorescence method for biomass measurements in algal growth inhibition tests. Water Res. 31, 2525-2531. doi:10.1016/\$0043-1354(97)00084-5 
Mayer, P., Holmstrup, M., 2008. Passive dosing of soil invertebrates with polycyclic aromatic hydrocarbons: Limited chemical activity explains toxicity cutoff. Environ. Sci. Technol. 42, 7516-7521. doi:10.1021/es801689y

Mayer, P., Nyholm, N., Verbruggen, E.M.J., Hermens, J.L.M., Tolls, J., 2000. Algal growth inhibition test in filled, closed bottles for volatile and sorptive materials. Environ. Toxicol. Chem. 19, 2551-2556. doi:10.1002/etc.5620191022

Mayer, P., Reichenberg, F., 2006. Can highly hydrophobic organic substances cause aquatic baseline toxicity and can they contribute to mixture toxicity? Environ. Toxicol. Chem. 25, 2639-2644. doi:10.1897/06142R.1

Mayer, P., Wernsing, J., Tolls, J., De Maagd, P.G.J., Sijm, D.T.H.M., 1999. Establishing and controlling dissolved concentrations of hydrophobic organics by partitioning from a solid phase. Environ. Sci. Technol. 33, 2284-2290. doi:10.1021/es9808898

OECD, 2011. Organisation for Economic Co-operation and Development. Freshwater Alga and Cyanobacteria, Growth Inhibition Test. OECD Guidelines for the testing of chemicals 201. doi:10.1787/9789264203785-en

OECD, 2000. Organisation for Economic Co-operation and Development. Guidance document on aquatic toxicity testing of difficult substances and mixtures. OECD Environmental Health and Safety Publications, Series on Testing and Assessment No. 23. doi:ENV/JM/MONO(2007)10

Panagopoulos, D., MacLeod, M., 2018. A critical assessment of the environmental fate of linear and cyclic volatile methylsiloxanes using multimedia fugacity models. Environ. Sci. Process. Impacts 20, 183-194. doi:10.1039/C7EM00524E

Reichenberg, F., Mayer, P., 2006. Two complementary sides of bioavailability: accessibility and chemical 

activity of organic contaminants in sediments and soils. Environ. Toxicol. Chem. 25, 1239-1245. doi:10.1897/05-458R.1

Rufli, H., Fisk, P.R., Girling, A.E., King, J.M.H., Länge, R., Lejeune, X., Stelter, N., Stevens, C., Suteau, P., Tapp, J., Thus, J., Versteeg, D.J., Niessen, H.J., 1998. Aquatic toxicity testing of sparingly soluble, volatile, and unstable substances and interpretation and use of data. Ecotoxicol. Environ. Saf. 39, 72-77. doi:10.1006/eesa.1997.1612

Schmidt, S.N., Smith, K.E.C., Holmstrup, M., Mayer, P., 2013. Uptake and toxicity of polycyclic aromatic hydrocarbons in terrestrial springtails-studying bioconcentration kinetics and linking toxicity to chemical activity. Environ. Toxicol. Chem. 32, 361-369. doi:10.1002/etc.2051

Seiler, T.B., Best, N., Fernqvist, M.M., Hercht, H., Smith, K.E.C., Braunbeck, T., Mayer, P., Hollert, H., 2014. PAH toxicity at aqueous solubility in the fish embryo test with Danio rerio using passive dosing. Chemosphere 112, 77-84. doi:10.1016/j.chemosphere.2014.02.064

Smith, K.E.C., Dom, N., Blust, R., Mayer, P., 2010a. Controlling and maintaining exposure of hydrophobic organic compounds in aquatic toxicity tests by passive dosing. Aquat. Toxicol. 98, 15-24. doi:10.1016/j.aquatox.2010.01.007

Smith, K.E.C., Oostingh, G.J., Mayer, P., 2010b. Passive dosing for producing defined and constant exposure of hydrophobic organic compounds during in vitro toxicity tests. Chem. Res. Toxicol. 23, 55-65. doi:10.1021/tx900274j

Smith, K.E.C., Rein, A., Trapp, S., Mayer, P., Karlson, U.G., 2012. Dynamic passive dosing for studying the biotransformation of hydrophobic organic chemicals: Microbial degradation as an example. Environ. Sci. Technol. 46, 4852-4860. doi:10.1021/es204050u 
non-toxic chemicals: "solubility addition" increases exposure for solid hydrophobic chemicals. Environ.

473 Stibany, F., Schmidt, S.N., Schäffer, A., Mayer, P., 2017. Aquatic toxicity testing of liquid hydrophobic $474 \quad$ chemicals - Passive dosing exactly at the saturation limit. Chemosphere 167, 551-558.

475 doi:10.1016/j.chemosphere.2016.10.014

476 Vergauwen, L., Schmidt, S.N., Stinckens, E., Maho, W., Blust, R., Mayer, P., Covaci, A., Knapen, D., 2015. A high throughput passive dosing format for the Fish Embryo Acute Toxicity test. Chemosphere 139, 917. doi:10.1016/j.chemosphere.2015.05.041 